Series A

I. MATHEMATICA

545

\title{
BOUNDARY MAPPINGS OF GEOMETRIC ISOMORPHISMS OF FUCHSIAN GROUPS
}

BY

TAPANI KUUSALO

H E L S I N K I 1973

S U OMALAINEN TIEDEAKATEMIA

https://doi.org/10.5186/aasfm.1973.545 
Copyright (C) 1973 by Academia Scientiarum Fennica ISBN-951-41-0113-8

Communicated 9 April 1973 by Olli Lehto 


\section{Boundary mappings of geometric isomorphisms of Fuchsian groups}

The object of the present paper is to apply certain ergodic theoretical results of E. Hopf ([2], [3]) to the study of boundary mappings of geometric isomorphisms of Fuchsian groups.

1. An isomorphism $j: G_{1} \rightarrow G_{2}$ of two Fuchsian groups acting in the unit disc $D=\{z \in \mathbf{C}:|z|<1\}$ is said to be geometric if there exists a homeomorphism $\Phi: D \rightarrow D$ inducing the isomorphism $j$, i.e. if we have

$$
\Phi \circ g=j(g) \circ \Phi
$$

for all $g \in G_{1}$. If both groups $G_{1}, G_{2}$ are the first kind, then $\Phi$ has a unique homeomorphic extension $\hat{\Phi}: \bar{D} \rightarrow \bar{D}$, so that also the boundary mapping $\varphi=\left.\hat{\Phi}\right|_{B d D}$ satisfies

$$
\varphi \circ g=j(g) \circ \varphi, g \in G_{1} .
$$

Unlike $\Phi$, the homeomorphism $\varphi: \mathbf{T} \rightarrow \mathbf{T}$ of the unit circle $\mathbf{T}=B d D$ is uniquely determined by the isomorphism $j$ ([5] $\S 3,[6] 3 . \mathrm{B})$. In the following, all Fuchsian groups are supposed to be of the first kind.

Occasionally we may study Fuchsian groups which act in the upper half plane $H$ instead of $D$. In that case we assume that the boundary mapping $\psi$ fixes the point $\infty$, so that $\psi$ will be a strictly monotone mapping $\psi: \mathbf{R} \rightarrow \mathbf{R}$.

2. We normalize the Lebesgue measure $\tau_{1}$ on $\mathbf{T}$ by $\tau_{1}(\mathbf{T})=1$, and the torus $\mathbf{T} \times \mathbf{T}$ has the product measure $\tau_{2}=\tau_{1} \times \tau_{1}$.

As a homeomorphism of the unit circle a boundary mapping $\varphi: \mathbf{T} \rightarrow \mathbf{T}$ has a derivative $\varphi^{\prime} \in \mathbf{C}$ a.e. on $\mathbf{T}$. Similarly a real-valued boundary mapping $\psi: \mathbf{R} \rightarrow \mathbf{R}$ which corresponds to Fuchsian groups acting in $H$ has a finite derivative $\psi^{\prime} \in \mathbf{R}$ a.e. on $\mathbf{R}$. Because $\psi$ is monotone, the derivative $\psi^{\prime}$ cannot change its sign.

Since the cross ratio $\left[z_{1}, z_{2}, z_{3}, z_{4}\right]$ is preserved under Moebius transformations it follows that also the differential

$$
d z_{1} d z_{2}\left(z_{1}-z_{2}\right)^{-2}=-\left[z_{1}, z_{2}, z_{1}+d z_{1}, z_{2}+d z_{2}\right]
$$


remains invariant. Let now $\varphi: \mathbf{T} \rightarrow \mathbf{T}$ be the boundary mapping corresponding to a geometric isomorphism $j: G_{1} \rightarrow G_{2}$. The invariance of (3) implies that also the expression

$$
\chi_{\varphi}\left(z_{1}, z_{2}\right)=\varphi^{\prime}\left(z_{1}\right) \varphi^{\prime}\left(z_{2}\right)\left[\frac{\varphi\left(z_{1}\right)-\varphi\left(z_{2}\right)}{z_{1}-z_{2}}\right]^{-2}
$$

is invariant under Moebius transformations. Thus if $h, k$ are two Moebius transformations, we have

$$
\chi_{亏}\left(k\left(z_{1}\right), k\left(z_{2}\right)\right)=\chi_{\varphi}\left(z_{1}, z_{2}\right)
$$

for $\xi=h \circ \varphi \circ k^{-1}: k \mathbf{T} \rightarrow h \mathbf{T}$. Since $G_{1}$ and $G_{2}$ have conjugate groups acting in $H$, we see that $\chi_{\varphi}: \mathbf{T} \times \mathbf{T} \rightarrow \mathbf{R}$ is a non-negative measurable function. Further it follows from (2) that $\chi_{\varphi}$ is automorphic with respect to $G_{1}$; that is,

$$
\chi_{\varphi}\left(g z_{1}, g z_{2}\right)=\chi_{\varphi}\left(z_{1}, z_{2}\right)
$$

for all $g \in G_{1}$.

3. The class $O_{H B}$. Suppose that the Riemann surface $S=D / G$ corresponding to a Fuchsian group $G$ is of class $O_{H B}$, i.e. $S$ does not have non-constant bounded harmonic functions, or equivalently that there is no non-constant $G$-automorphic bounded harmonic function in $D$. Using the Poisson representation we see that all $G$-automorphic bounded harmonic functions are constant if and only if the action of $G$ on $\mathbf{T}$ is metrically transitive, i.e. if and only if a measurable $G$-invariant subset $E \subset \mathbf{T}$ has either measure $\tau_{1}(E)=0$ or $\tau_{1}(E)=1$.

Theorem 1. Let $\varphi$ be the boundary mapping of a geometric isomorphism $j: G_{1} \rightarrow G_{2}$. If one of the Riemann surfaces $S_{i}=D G_{i}, i=1,2$, is of class $O_{H B}$, then the mapping $\varphi$ is either absolutely continuous or completely singular.

Proof. Suppose that $S_{2}$ is of class $O_{H B}$. If \& is not absolutely continuous, there exists a Borel set $E \subset \mathbf{T}$ such that $\tau_{1}(E)=0, \tau_{1}(q(E))>0$. The set $F_{1}=G_{1} E$ is invariant under $G_{1}$; and $F_{2}=q\left(F_{1}\right)=G_{2} q(E)$ under $G_{2}$. Now $\tau_{1}\left(F_{1}\right)=0$, and $\tau_{1}\left(F_{2}\right)=1$ since $G_{2}$ is metrically transitive. Thus both $\varphi$ and $\varphi^{-1}$ are completely singular.

4. The Hopf classification. Let $S$ be a hyperbolic Riemann surface, $T(S)$ the tangent manifold of $S$, and $\sigma_{x}(v, w), x \in S, v, w \in T_{x}(S)$, the hyperbolic metric of $S$. Since $S$ is a complete Riemannian manifold with respect to the hyperbolic metric, the geodesic flow $\beta_{t}$ determined 
by the Lagrangian $L(x, \dot{x})=\sigma_{x}(\dot{x}, \dot{x})$ is globally defined on $T(S)$, i.e. $\beta_{t}: T(S) \rightarrow T(S), t \in \mathbf{R}$, is a one-parameter transformation group. The surfaces $\mathscr{E}_{c} \subset T(S)$ of constant energy, $L(x, v)=c$, are invariant under the geodesic flow, and since the flow $\beta_{t}$ is essentially similar on every $\mathscr{E}_{c}, c>0$, we can consider only $\mathscr{E}=\mathscr{E}_{1}$. The geodesic flow $\beta_{t}$ restricted to $\mathscr{E}$ is simply the flow of unit speed along geodesics.

E. Hopf has shown that the geodesic flow $\beta_{t}$ of a hyperbolic Riemann surface $S$ always is either ergodic or dissipative on $\mathscr{E}$ ([2], [3]). The surface $S$ is said to be of the first class in the ergodic case, and of the second class in the dissipative case. Suppose now that the surface $S$ is represented by a Fuchsian group $G$ acting in $D, S=D / G$. It follows then further that $S$ is of the first class if and only if the action

$$
\{g,(x, y)\} \mapsto(g x, g y), g \in G, \quad(x, y) \in \mathbf{T} \times \mathbf{T},
$$

of $G$ on the torus $\mathbf{T} \times \mathbf{T}$ is metrically transitive, i.e. if and only if each measurable $G$-invariant subset $E \subset \mathbf{T} \times \mathbf{T}$ has either measure $\tau_{2}(E)=0$ or $\tau_{2}(E)=1$ ([2] 8.1). It follows immediately that every surface of the first class is always of class $O_{H B}$.

Theorem 2. Suppose that one of the Riemann surfaces $S_{i}=D / G_{i}$, $i=1,2$, is of the first class. Then for each geometric isomorphism $j: G_{1} \rightarrow G_{2}$ either the boundary mapping $\varphi$ is completely singular or the isomorphism is induced by a Moebius transformation on $\mathbf{T}$.

Proof. Let $S_{1}$ be of the first class, so that the boundary mapping is either absolutely continuous or completely singular by the preceding theorem. Since $\chi_{\varphi}$ is $G_{1}$-automorphic by (6), it is equal to a constant a.e. on $\mathbf{T} \times \mathbf{T}$. Obviously we must have $\chi_{\varphi}=1$ a.e. in the case of absolute continuity, and $\chi_{\varphi}=0$ a.e. in the singular case.

Suppose now that $\varphi$ is absolutely continuous. Using appropriate Moebius transformations $h, k$ we can find groups $G_{1}^{\prime}=h G_{1} h^{-1}, G_{2}^{\prime}=$ $k G_{2} k^{-1}$ acting in $H$ with a real-valued boundary mapping

$$
\psi=k \circ \varphi \circ h^{-1}: \mathbf{R} \rightarrow \mathbf{R} .
$$

We may further suppose that $\psi(0)=0, \psi^{\prime}(0)=1$, so that $\psi$ satisfies on $\mathbf{R}$ the differential equation

$$
\psi^{\prime}(x)=\psi(x)^{2} / x^{2}
$$

because $\psi_{\psi}=1$ a.e. on $\mathbf{R} \times \mathbf{R}$. But given the initial value $\psi(0)=0$, $\psi(x)=x$ is the only solution of (8) continuous on all of $\mathbf{R}$. Thus $\varphi=k^{-1} \circ h$, so that the isomorphism $j$ is induced on $\mathbf{T}$ by a Moebius transformation. 
5. A Riemann surface $S=D / G$ can obviously be of the first class only if $G$ is a Fuchsian group of the first kind, but this condition is by far insufficient. If $S \subset \hat{\mathbf{C}}$ is a hyperbolic planar surface, the covering group of $S$ is of the first kind if the complement $\hat{\mathbf{C}} \backslash S$ is totally disconnected, but $S$ is of class $O_{H B}$ if and only if $\widehat{\mathbf{C}} \backslash S$ has vanishing logarithmic capacity.

If $A$ is the hyperbolic area of a hyperbolic Riemann surface $S$, the volume of $\mathscr{E}$ is $2 \pi A$ (cf. n:o 4), so that all Riemann surfaces of finite hyperbolic area are of the first class by Poincaré's recurrence theorem ([2] $7.1,[3])$. Now the hyperbolic area of a Riemann surface $S=D / G$ is finite if and only if $G$ is a finitely generated group of the first kind ([4], Theorem 5). Thus the Riemann surface $S=D / G$ is of the first class for all finitely generated Fuchsian groups $G$ of the first kind.

Theorem 3. Suppose that the geometric isomorphism $j: G_{1} \rightarrow G_{2}$ of two finitely generated Fuchsian groups of the first kind acting in $H$ has an increasing boundary mapping $\psi: \mathbf{R} \rightarrow \mathbf{R}$. Then $\psi$ is either affine or a completely singular quasisymmetric function.

Proof. If $G$ is a finitely generated Fuchsian group of the first kind, the Riemann surface $S=\left(S_{G}, n_{G}\right)=H / G$ is a pointed surface of finite type, i.e. $S$ is a compact surface $S^{\prime}$ with finitely many punctures; further, the support of $n_{G}$ is finite. Thus in the case of finitely generated groups of the first kind there always exists a quasiconformal mapping $\Phi: H \rightarrow H$ inducing the given isomorphism $j$ (cf. [5] Theorem 2.1, [6] 2.B), so that the boundary mapping $\psi: \mathbf{R} \rightarrow \mathbf{R}$ must be quasisymmetric, and the conclusion follows now from theorem 2 .

Recently Sorvali has obtained results of a similar kind (cf. [5] Theorem 5.1). For quasisymmetric functions, cf. also Beurling - Ahlfors [1], for singular functions especially $\S 7$.

Institut Mittag - Leffler

Djursholm, Sweden
Current address:

University of Jyväskylä Jyräskılä, Finland 


\section{References}

[1] Beurling, A., and L. Ahlfors: The boundary correspondence under quasiconformal mappings. - Acta Math. 96, 125-142 (1956).

[2] Hopf, E.: Statistik der geodätischen Linien in Mannigfaltigkeiten negativer Krümmung. - Ber. Verh. Sächs. Akad. Wiss. Leipzig 91, 261-304 (1939).

[3] -»- Ergodic theory and the geodesic flow on surfaces of constant negative curvature. - Bull. Amer. Math. Soc. 7\%, 863-877 (1971).

[4] Sieged, C. L.: Some remarks on discontinuous groups. - Ann. of Math. 46, $708-$ $718(1945)$.

[5] Sorvali, T.: The boundary mapping induced by an isomorphism of covering groups. - Ann. Acad. Sci. Fenn. A I 526, 1-31 (1972).

[6] Tukia, P.: On discrete groups of the unit disk and their isomorphisms. - Ann. Acad. Sci. Fenn. A I 504, 1-45 (1972). 